\title{
DESIGNING AN ONLINE SELF-ASSESSMENT TOOL UTILIZING CONFIDENCE MEASUREMENT
}

\author{
GRAHAM FARRELL ${ }^{1}$ and YING K. LEUNG $^{2}$ \\ ${ }^{I}$ School of Information Technology, Swinburne University of Technology, Australia \\ ${ }^{2}$ Department of Information \& Communications Technology, Hong Kong Institute of \\ Vocational Education
}

Abstract: The convergence of multi-disciplined technologies to address and support the rapid evolution of e business is critical to the success of many ventures. There is an increasing demand for the availability of tools to assist in the evaluation and processing of information as it flows between the bodies involved. A tool that could predict and forecast expected occurrences with a stated level of confidence is considered to be extremely valuable and well worth pursuing. The area of Web based learning is rapidly becoming a fundamental component of corporate training areas and educational Institutions, with an increasing reliance on the delivery of their programs via the Internet or Intranet. The success of such programs is dependent on the marrying of Internet technology, Information systems, Human Computer Interaction and Educational Strategies while catering for the individual student.

This research investigates and evaluates the design of an innovative approach to a formative assessment that permits the participant to register their level of confidence. The system then provides enriched feedback and can predict the performance of the individual, reducing the number of failures. The system is based on the traditional Online Multiple Choice Questions format, but it facilitates a method of registering the level of confidence of the student's response to each of the options available, generating a score that reflects their level of understanding of the topic being considered. The iterative process of design and the results of a pilot program are discussed, and directions for further studies suggested.

The original version of this chapter was revised: The copyright line was incorrect. This has been corrected. The Erratum to this chapter is available at DOI: 10.1007/978-0-387-35692-1_36 


\section{INTRODUCTION}

E-learning has in recent times become a major commercial enterprise. The supplying of quality education, at a global level, at a reasonable cost and with the additional convenience to the client, is seen to be a profitable opportunity. The cost efficiency of e-learning in the corporate sector is potentially so large that many substantial companies around the world are developing online materials for their in-house training or negotiating with established online education providers to do the same. In addition to this other organizations have established profitable business through the onselling of material that they have developed.

Assessment plays a critical role in the educational process as both a means of grading and supplying valuable feedback to the students. The embracing of technology as an integral part of the delivery of education has increased the requirement for effective assessment systems to be available to the student, encouraging self-assessment at all stages of the learning experience. It is accepted wisdom that testing for the purpose of feedback should be an integral part of the sequence of learning activities rather than an interruption to that sequence. See Principles and Standards for School Mathematics (2000) for example.

In view of the commercial importance of e-learning, and the fundamental role played by assessment in the learning process, it is essential that we explore technologies and approaches that will improve assessment effectiveness in e-learning and its impact on the learning process. The elearning removes the personal interactivity held within the classroom situation. The challenge for e-learning is to enhance the online learning experience with techniques that provide rich, personalized feedback to the individual student.

Traditionally both formative and summative methods of assessment have been reliant on the instructor to supply the feedback. With the everincreasing demand to constrain delivery costs, resulting in increased student staff ratios, unintentional delays in supplying feedback to the student can occur to the disadvantage of both the student and the instructor. As a result often the most valuable feedback occurs at the final stages of the learning path generally too late to be of great value to the students' learning process and providing only limited feedback to the instructor. This situation tends to benefit primarily the group of students participating in the next scheduled delivery of the unit. To maximize the effect and value of feedback it must be instantaneous, an inherent characteristic of an online assessment tools due to the nature of the encompassing technology. The formative assessment feedback cycle should occur early, and then constantly throughout the duration of the learning experience. 
Doebbert (1999) emphasizes the need for the student to develop skills in managing and controlling their learning, with the utilization of technology assisting in the process as they travel the educational path. Accordingly we are interested in looking at approaches to assessment, which help to place control of the learning in the hands of the student

This paper proposes and investigates the design of an innovative formative assessment tool, Multiple Choice Questions Confidence Monitor (MCQCM), using internet technology. The objective of the project is to develop a self-assessment system that provides enriched feedback to both the student and the instructor in order to benefit all those associated with the learning process. The self-assessment package is based on traditional Multiple Choice Questions. However it utilizes a method of students' registering their level of confidence for each of the options available. The package instantly generates a score that aims to reflect the students' level of understanding of the topic being considered. We discuss the initial design considerations and the corresponding pilot program that followed.

\section{FORMATIVE ASSESSMENT}

Black and William (1998) use the term "Assessment" as referring to the group of activities undertaken by both teachers and students in assessing themselves, providing both grades and feedback to modify teaching. They state that " such assessment becomes formative assessment when the evidence is actually used to adapt the teaching to meet the students needs". On the other hand it is generally accepted that summative assessment is a tool that has the primary objective of supplying a grade for the student.

Black and William (1998) refer to the learning environment as being a "Black Box", with input from pupils, teachers, parents and resources, with the consequential outputs being pupils with advanced educational standing. It is within this Black Box that they consider the role of Formative Assessment critical in the transformation of the students' educational standing, contributing to the raising of the national standards.

Marshall University (1999) provides a thorough comparative study of online course delivery software, identifying the active components of each package and the depth of their application. Not surprisingly, online assessment utilizing multiple choice questions format appears in all of the recommended packages such as Blackboard, WebCT, TopClass, Web Course in a Box, Toolbook, and others. They further state that only five of the identified ten packages that offer formative assessment also provide an 
effective feedback mechanism that directs the student to tutorial paths as a consequence of formative assessment.

Black and William (1998) sate that pupils too often " are content to get by". Perrenoud (1991) suggests that a solution would be for the instructors to "reconstruct the teaching contracts to counteract these acquired habits" with the inclusion of formative assessment as a key component of the learning process.

Resulting amended instructional paths instigated from formative assessment is critical for the students' success in navigating the learning path. We consider instruction and formative assessment not to be just strongly linked but inseparable components in the learning experience.

\section{MULTIPLE CHOICE QUESTIONS AND THE ONLINE ENVIRONMENT}

The general acceptance of the multiple choice question (MCQ) formats as both a formative and summative assessment tool has seen it extensively utilized in traditional education. This is mainly due to the ability of MCQs to assess a broad field of learning in a compact system that is quick to assess and offers student feedback without excessive cost. The ease of the adaption of the MCQ format to the Hypermedia environment produced fully integrated and automated systems, supplying instant feedback to the students' with suggested direction for further study.

MCQs offer an excellent method of assessing the students knowledge in areas deemed as either right or wrong. However, what about the "shades of gray" or fuzzier areas? Diamond and Forrestor (1973) define knowledge as asking the question "What do you know?" followed by the meta-question of equal importance "How sure are you of the answer to the question about what you know?".

Davidoff (1995) recognizes the need for a more thorough approach in assessing students in the medical arena. He suggests that a system designed to recognize "incomplete or partial knowledge", permitting the student to "hedge", would be greatly beneficial to the learning process. He further states that much of the medical knowledge is "incomplete, ambiguous and conflicting" and that the standard MCQ testing method does not facilitate or reflect the students' level of knowledge and their confidence in that level of knowledge. One of his main concerns is that MCQs recognize and reward those areas of knowledge that is either right or wrong, encouraging guessing and often leading to overconfidence, and he considers "miss-calibrated confidence" in medical education equally as concerning as lack of knowledge. 


\section{Measurement}

The scoring of MCQs has long been a point of discussion with serious consideration been given to various scoring models. Consequential scoring models designed for the purpose of eliminating guessing utilize complex scoring algorithms that aim to produce a reflection of the students understanding of the underlying concepts.

Brown and Shuford (1973) produced an MCQ calibrated scoring system "that encouraged honesty", designed to reflect the level of confidence in choosing the answer whilst severely penalizing the participant if they answered incorrectly. The primary objective of this scoring system is to identify students that are either overconfident or under confident.

Pollard and Clarke (1989) produced alternative MCQ scoring techniques designed to minimize random guessing due to the reward and penalty structure of the scoring system. Various institutions have used these methods however, to be effective, full understanding of the calculations involved in the grading schedule is required.

MCQs have traditionally required the student to identify the correct response from a list of possible answers. Traditionally the resulting score is based on the criteria of being correct or incorrect. Pollards (1985, 1986, 1993) extensive scoring mechanism aims to eliminate guessing by allocating a mark for each correct box ticked and each incorrect box ticked.

We see merit in this system but have concerns with the level of complexity in operation by the participant. The student must fully comprehend the consequences of his/her action. The system relies on the combination of boxes ticked with many variations that have to be considered.

Klinger (1997) utilized a system of scoring where the student nominated a position on lines joining the apexes in a triangular shape. Each of the apexes represented the three optional answers. He proportionally divided the lines joining the apexes into segments encouraging the student to nominate their level of confidence. The grade was then scored according to a graduated linear scale of logarithms, rewarding the student if they chose to declare that they were completely unsure of their answer.

This is also the case with other designed systems such as Paul's (1994) interactive response system that requires the student to choose an option demonstrating a level of confidence in their answer. With this system the student places the cursor within a grid area aimed to reflect the confidence of their choice. The system is presented to the student in the format shown in Fig 1. The student must negotiate the area with a mouse and click on the region that they feel portrays their confidence. Each of the three options are located at the vertices of the triangle and the closer the student positions the cursor to the vertex the more confident they are that it is the correct answer. 
A corresponding score is then calculated. We have some initial concerns that this system could be difficult to use, as the regions appear to be cluttered at the vertices, and the scoring system for a centrally located choice of no commitment (.33 on the diagram) to any option scores a high 63 .

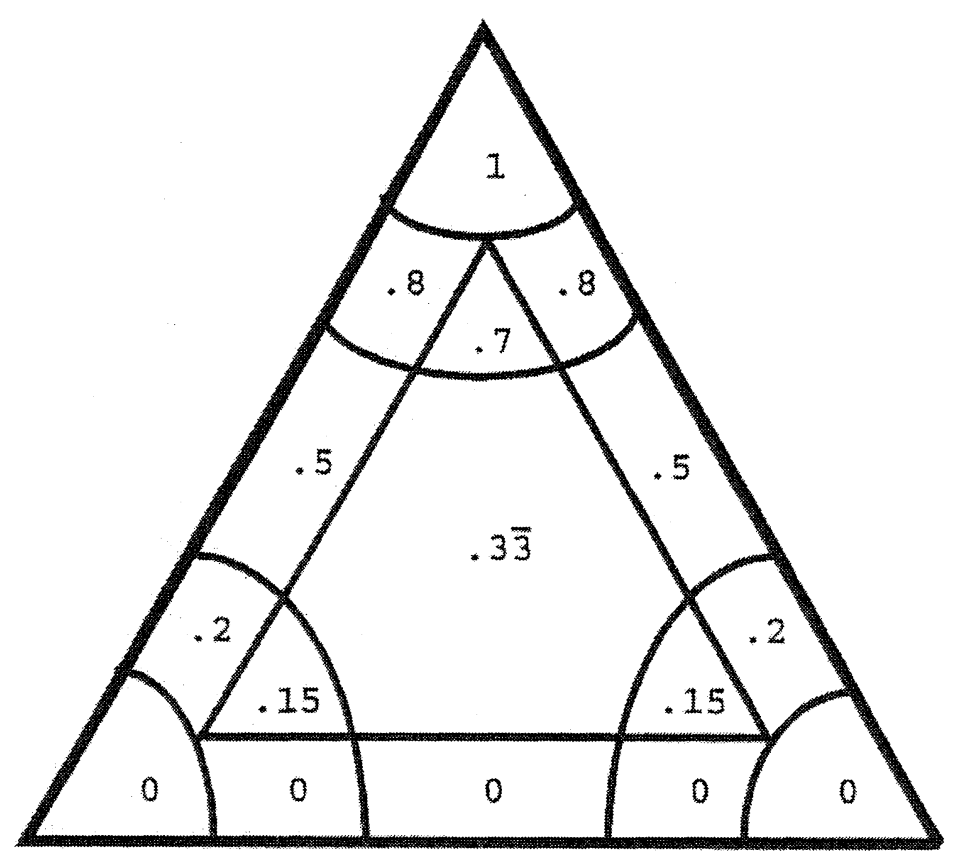

Figure 1. Confidence Measuring Template where the apex is the correct answer showing the consequential values awarded (Paul 1994)

We also consider the user interface to be difficult as it calls upon extreme cognitive load to be operable to a large group of participants.

Previous research has provided a good foundation for the development of formative assessment tools to the benefit of all concerned. The research of Pollard (1989) demonstrates the advantages of developing a scoring system that aims to reflect the students' understanding of the subject while Paul (1994), Brown and Shufford (1993) and Klinger (1997) all have demonstrated variations on systems designed to capture a numerical representation of the students level of knowledge.

The combination of this previous research with the rapid development of the Internet as a means of educational delivery and assessment encourages the designing, testing and evaluation of formative assessment tools that 


\section{Measurement}

incorporate scoring systems aimed to reflect the level of confidence of the student, keeping them honest to themselves for their benefit.

\section{THE MCQCM SELF-ASSESSMENT TOOL}

The MCQCM is based on the traditional Multiple Choice Questions format, consisting of a stem with three or four options, as recommend by Kehoe (1995) and Frary (1995). The MCQCM is scalable to permit a variation on the available options as Frary (1995) states that the number of options can be restricted by the available sensible solutions.

In designing the MCQCM the following further criteria were considered, that is the self-assessment tool should:

- Be easy to use and not too demanding of the user.

- Utilize a scoring technique that is simple, placing the user in control of the consequences of their actions.

- Have good usability, engaging the user while exercising sound navigational properties for delivery across the Internet.

- Be able to capture and record the scores of the students as they participated in the exercise.

The final version of the MCQCM used for the trials was a result of considering all of the points culminating in the working prototype with the features described below.

The MCQCM:

- Permits more than one correct answer. This encourages the student to consider all options separately and not to identify what they consider to be the single correct answer and ignore the rest. The advantages of this system above the traditional MCQ format are as follows:

- Permits the instructor to word the options to closely examine the areas of study, eliminating the need to use easily recognizable distracters.

- Forces the student to consider all options carefully, increasing their exposure to associated areas within the topic.

The MCQCM was required to produce a score aimed to reflect the students' understanding of the concept being considered in each question. A simple scoring technique, easily understood by the student, gave the student control. Consequently, the students' were not surprised by the responses of the MCQCM and the cognitive load upon the user was minimized.

Each of the options within a question is scored separately on a continuum from -10 to 10 , giving feedback for both correct and incorrect answers. If an option was True and the student moved the slide bar to the right towards the 
True label then they would be rewarded with a positive grade depending on their recorded level of confidence. If the recorded level of confidence was $100 \%$ then the resulting score would be 10 marks, and so on for each decrement of $10 \%$.

The same scoring system applies for the situation where the option was False and the student moved the slide bar to the left towards the False label. The position of the slide bar would be recorded and the score would be a positive representation of the level of confidence, being 10 marks for $100 \%$ level of confidence. All positive scores were shown in Black.

If an option was True and the student moves the sliding bar to the left towards the False label then the student receives a negative score directly proportional to their recorded level of confidence. If the position is $100 \%$ the corresponding score is -10 and so on for decrements of $10 \%$. The same scoring system applies to a situation where the option was False and the student moved the sliding bar towards the True label. All negative scores were shown in Red to ensure that the student realized that their response was incorrect. The final score for each option was given to the student after the completion of the test along with the correct answers.

This MCQCM is designed to reflect the students' level of understanding. The students' are placed in the position where they must clearly state their level of confidence, knowing that they would be proportionally penalized for an incorrect choice and proportionally rewarded for a correct choice. The MCQCM also permits the use of questions that have multiple correct answers, which requires them to identify all possible correct combinations and score accordingly. The design of the MCQCM is shown in Fig 2 . 


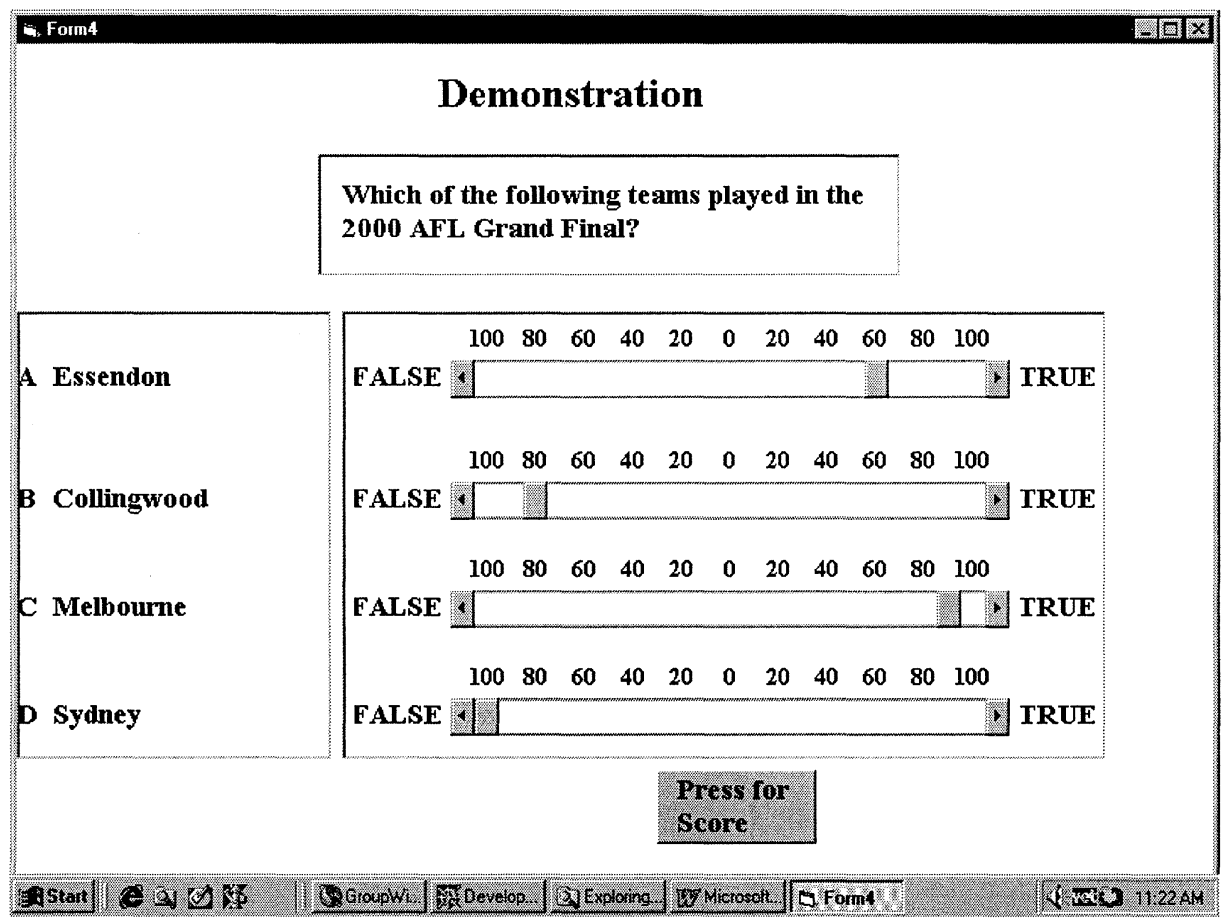

Figure 2. The MCQCM showing the demonstration screen used as part of the training

\section{PILOT STUDY}

During the development of the working prototype, discussions with experienced instructors about the functionality and design of the MCQCM identified the need to run an initial pilot program. Our objective was to elicit feedback from the operation of the prototype system.

We used Visual Basic to construct the interface with the ability to create individual data tables for each participant. The test responses from each of the participants were captured and displayed to them on completion of the exercise. 
In keeping with recommendations for usability testing three males and three female students were invited to complete a test using the newly developed tool in the presence of the designers. The test consisted of five questions pertaining to an area of their study due to be formally assessed. Consequently the students perceived the test as advantageous to their study and were keen to participate.

To help the students become familiar to the self-assessment MCQCM they all participated in an introductory session that demonstrated the package in a non-threatening way. The students were initially required to respond to a general knowledge question set in their social environment. This question addressed a major, well-publicized, sporting event. After successful completion they started the formal test.

The subjects were all observed for the duration of the pilot program and the observations recorded. In addition they were also encouraged to verbalize their thought processes (Verbal Protocol) as they navigated the path of the test. On completion of the test they all participated in an interview session where they were asked a series of questions.

- Was the MCQCM easy to operate?

- Did you understand the scoring system that penalized for incorrect choices and rewarded for correct choices?

- Did you actively use the sliding bar to register your level of confidence freely?

- Did you understand the feedback display produced?

- Do you think that the feedback and resulting score would help you decide your study path?

\section{OBSERVATIONS AND RESULTS}

The initial introductory session required the subjects to use the tool in a non-threatening environment. This proved to be a successful exercise as they responded to the MCQCM in a relaxed manner. The students were then requested to complete the more formal part of the pilot program. It was observed that all students completed the test without any real operational concerns. However, despite the successful introductory session, there was still some initial apprehension in using the MCQCM.

It was apparent from their verbal protocol during testing they were not all together comfortable with the interface, due to the lack of familiarity. They also expressed concerns about being required to identify not only what they consider to be the correct options but also what they consider to be the incorrect ones. Some demonstrated hesitation registering their level of 
confidence in all of the options, but tended to only do so for the correct options.

The simple scoring system was well received. During the interview all of the subjects claimed to understand the method of calculating the score and consequently would react depending on their level of confidence to maximize their result. Further, all of the subjects stated during the interview that they understood the feedback, as it clearly reflected their responses. They also agreed that that the feedback would assist them in deciding their study path to improve their understanding of the topic.

It was observed, and confirmed during the interview, that the students tended to minimize the use of the slide bar for the first few questions. However, as the student became more relaxed with using the MCQCM, the use of varying levels selected on the slide bar increased.

All of the students requested for more opportunities to use the test as they considered it to be greatly beneficial in confirming their knowledge and highlighting their weaknesses in the topic covered.

\section{DISCUSSION AND LIMITATIONS}

The MCQCM was well received by all of the participants in the pilot program. The students appreciated the opportunity to use the self-assessment MCQCM and considered it beneficial in their preparation for the oncoming assessment task.

The students considered the MCQCM easy to operate and the method of scoring appeared to encourage risk taking, resulting in the students manipulating the MCQCM to their advantage. They increasingly used the sliding bar to register their confidence and confirmed that the feedback and consequential score was both comprehensible and helpful for further study direction.

A limitation was that the pilot program was not broad enough to give us any valid feedback about the biasness towards particular learning styles and gender. The students could neither demonstrate nor comment on these issues during their short exposure.

\section{FURTHER WORK}

The cognitive process of decision-making is to be explored to see if the choice of the option being either true or false is dominant in the participants mind, or if the expression of the level of confidence is the dictating factor. In 
simple terms, "is stating that you are $30 \%$ sure that the answer is false the same as saying that you are $70 \%$ sure that the answer is true?". These questions still need to be investigated and further trials completed in order to develop a tool to maximize the benefit to the students.

We intend on conducting a wider study to determine if the MCQCM is biased towards particular learning styles or groups of learners. Feedback would also be welcome on improvements or usability aspects of the interface aimed to improve the usability and thereby enhance the students' learning path.

An opportunity exists to apply the MCQCM to various other arenas that would benefit from the Web based technology, particularly e-comerce. Jamieson (1998) explored the application of an automated auditing tool (KASE) with great success that permitted the user to register their level of confidence of a particular situation being susceptible to fraud. An area of interest could be in registering consumer trust indexes.

\section{REFERENCES}

Black. P and William D (1998): Inside the Black Box: Raising Standards Through Classroom Assessment. Phi Delta Kappan October 1998 Volume 80 Number 2 P 139-149 http://www.pdkintl.org/kappan/kbla9810.htm

Black. P and William D (March 1998): Assessment and Classroom. Learning Assessment in Education, Vol 5 March P. 7-74

Brown TA and Shuford EH (1973): Quantifying Uncertainty Into Numerical Probabilities for the Reporting of intelligence. Defence Advanced Research Projects Agency Report R1185-ARPA. Santa Monica, Calif., Rand Corp

Davidoff F (Feb 1995): Confidence Testing- How to Answer a Meta-Question. American College of Physicians Observer www.acponline.org/journals/news/feb95/conftest.htm

Diamond GA, and Forrester JS (1983): An Epistemologic Model of Clinical Judgment. Am J Med 75: P129-137

Doebbert J (1999): Benchmarking the Learning Environment (Technology). National Centre of Research in Vocational Education (Copa \& Ammentorp press) Chpt 5 vocserve.berkeley.edu/abstracts/MDS-1108/MDS-1108-CHAPTER-5.html

Frary R (1995): More multiple-choice item writing do's and don'ts. Practical Assessment, assessment and evaluation ERIC Clearinghouse on Assessment and Evaluation. Vol 4 (11) ericae.net/pare/getvn.asp? $\mathrm{v}=4 \& \mathrm{n}=11$

Jamieson, R. (1999): An Investigation of Auditor Decision-Making in an Information Systems Audit Domain, Unpublished PhD Dissertation, University of New South Wales.

Kehoe J (1995): Writing Multiple Choice Test Questions Practical Assessment, assessment and evaluation ERIC Clearinghouse on Assessment and Evaluation. Vol 4 (9) http://ericae.net/pare/getvn.asp? $=4 \& \mathrm{n}=9$

Klinger A (1997): Experimental Validation of Learning Accomplishment. Frontiers in Education. Technical Report No. 970019

Marshal University (1999): Comparison of Online Course Delivery Software Products. multimedia.marshall.edu/cit/webct/compare/comparison.html 
Paul J (1994): Improving Education Through Computer Based Alternative Assessment Methods. People and Computers Vol 9

Perrenoud P (1991): Towards a Pragmatic Approach to Formative Evaluation. Assessment of Pupils' Achievement: Motivation and School Success (Amsterdam: Swets and Zeitlinger) P. 92

Pollard G (1985): Scoring in Multiple Choice Examinations. Math. Scientists Vol 10 P 93-97

Pollard G (1986): Scoring to Remove Guessing in Multiple Choice Exams. Math Education Science Technology Vol 20 No2 P 33-36

Pollard G and Clark D (1989): An Optimal Scoring System of Multiple Choice Competitions and an Analysis of Candidates Responses Under Two Different Methods of Scoring. Mathematics Competitions Vol 2 No 2 P33-36

Pollard G (1993): Further Scoring Systems to Remove Guessing in Multiple Choice Examinations. Mathematics Competitions Vol 2 No 1 P 27-43

Principle and Standards for School Mathematics (2000): National Council of Teachers of Mathematics - Standards 2000 Project Chpt 2. 